\title{
Molecular dynamics and density functional studies of a body-centered-tetragonal polymorph of $\mathrm{ZnO}$
}

\author{
J. Wang, ${ }^{1}$ A. J. Kulkarni, ${ }^{2}$ K. Sarasamak, ${ }^{3}$ S. Limpijumnong, ${ }^{3}$ F. J. Ke, ${ }^{1,4}$ and M. Zhou ${ }^{2, *}$ \\ ${ }^{1}$ Department of Physics, Beihang University, Beijing 100083, China \\ ${ }^{2}$ School of Mechanical Engineering, Georgia Institute of Technology, Atlanta, Georgia 30332-0405, USA \\ ${ }^{3}$ School of Physics, Suranaree University of Technology, Nakhon Ratchasima 30000, Thailand \\ and National Synchrotron Research Center, Nakhon Ratchasima 30000, Thailand \\ ${ }^{4}$ Institute of Mechanics, Chinese Academy of Sciences, Beijing 100080, China \\ (Received 13 September 2007; published 13 November 2007)
}

\begin{abstract}
We report a previously unknown body-centered-tetragonal structure for $\mathrm{ZnO}$. This structure results from a phase transformation from wurtzite in [0001]-oriented nanorods during uniaxial tensile loading and is the most stable phase for $\mathrm{ZnO}$ when stress is above $7 \mathrm{GPa}$. The stress-induced phase transformation has important implications for the electronic, piezoelectric, mechanical, and thermal responses of $\mathrm{ZnO}$. The discovery of this polymorph brings about a more complete understanding of the extent and nature of polymorphism in $\mathrm{ZnO}$. A crystalline structure-load triaxiality map is developed to summarize the relationship between structure and loading.
\end{abstract}

\section{DOI: 10.1103/PhysRevB.76.172103}

Natural selection of the lowest energy state determines the bonding state and atomic arrangement of a material under ambient conditions. Deviations from this natural state occur when external stimuli such as mechanical loading and temperature changes are provided, leading to failure through bond breaking or polymorphism due to atomic rearrangement. At the macroscopic scale, failure is dominant since atomic mobility is relatively low and defects are more prevalent. At the nanoscale, however, high surface-to-volume ratios and nearly defect-free structures lead to higher atomic motilities and more pronounced polymorphic transitions. Consequently, polymorphs previously unknown for bulk materials can be revealed. Recently, a fivefold coordinated hexagonal phase (referred to as $\mathrm{HX}$ ) of $\mathrm{ZnO}$ was observed in [0110]-oriented $\mathrm{ZnO}$ nanowires under uniaxial tensile loading. ${ }^{1,2}$ This discovery has subsequently been confirmed in [0001]-oriented $\mathrm{ZnO}$ nanoplates ${ }^{3}$ and nanowires. ${ }^{4}$ Here, we report yet another polymorph of $\mathrm{ZnO}$ with a bodycentered-tetragonal structure with four-atom rings (referred to as BCT-4, space group $P 4_{2} / \mathrm{mnm}$ ), which occurs under uniaxial tensile loading along the [0001] crystalline axis of the wurtzite structure. While similar structures have been reported for carbon ${ }^{5}$ and lithium aluminum oxide, ${ }^{6,7}$ this polymorph has been reported here for a binary system. The results here show that the extent of polymorphism in $\mathrm{ZnO}$ (and perhaps in other groups IV, III-V, and II-VI materials such as $\mathrm{GaN}$ and $\mathrm{CdSe}$ ) is much more pronounced than previously known. With the discovery of these phases, a more complete picture has emerged for the polymorphism of $\mathrm{ZnO}$ under the influence of mechanical loading with all realistic triaxialities. The recent fabrication and applications of defect-free, single-crystalline nanowires, nanobelts, and nanorings of materials such as $\mathrm{ZnO}, \mathrm{GaN}$, and CdSe highlights the need for understanding the extent of polymorphism. Characterization of the thermomechanical and electrical responses of the relevant phases is crucial since the performance and functionalities of these slender quasi-onedimensional materials as components in ultrasensitive chemical and biological sensors, nanoresonators, field effect
ACS number(s): $61.50 . \mathrm{Ks}, 61.46 .-\mathrm{w}, 62.25 .+\mathrm{g}, 64.70 . \mathrm{Nd}$

transistors, and nanogenerators ${ }^{8-11}$ are either significantly affected by or utilize the phase transitions. ${ }^{12-14}$

Our analyses include both molecular dynamics (MD) simulations and density functional theory (DFT) based first principles calculations. The MD simulations are performed to study the phase transformation and the associated mechanical response of $\mathrm{ZnO}$ nanorods with the [0001] growth direction under loading and subsequent unloading. The first principles calculations are carried out to determine the energetic favorability and the electronic band structures of the parent and transformed phases. The impact of this phase transformation on the thermal, mechanical, and electric responses of the nanorods is also evaluated.

The as-synthesized hexagonal $\mathrm{ZnO}$ nanorods have a wurtzite structure with a sixfold symmetry around the [0001] axis and six $\{01 \overline{1} 0\}$ lateral crystalline surfaces, ${ }^{15,16}$ as illustrated in Fig. 1(a). The lattice parameters (Ref. 16) are $a$ $=3.25 \AA, u=0.38$, and $c=5.21 \AA$ as shown in Fig. 2(a). The

(a)
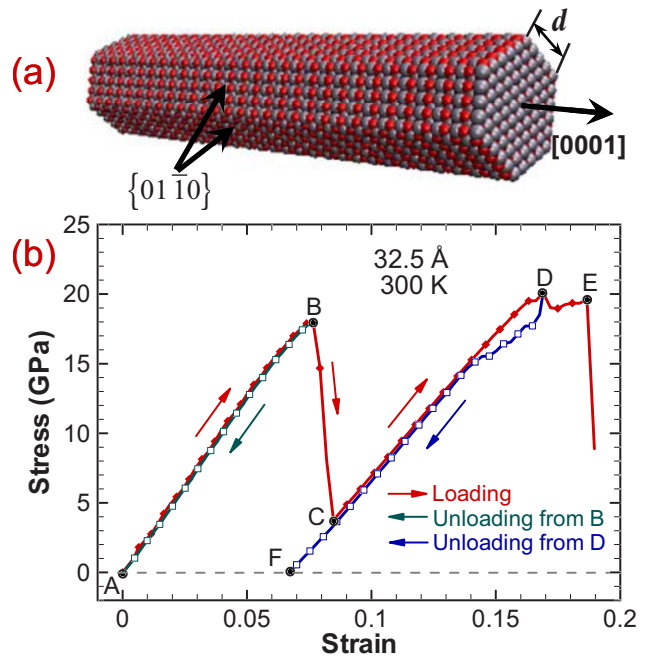

FIG. 1. (Color online) (a) [0001] nanorod with $d=32.5 \AA$ and (b) stress-strain curve of this nanorod at $300 \mathrm{~K}$ during loading and unloading. 
(a)
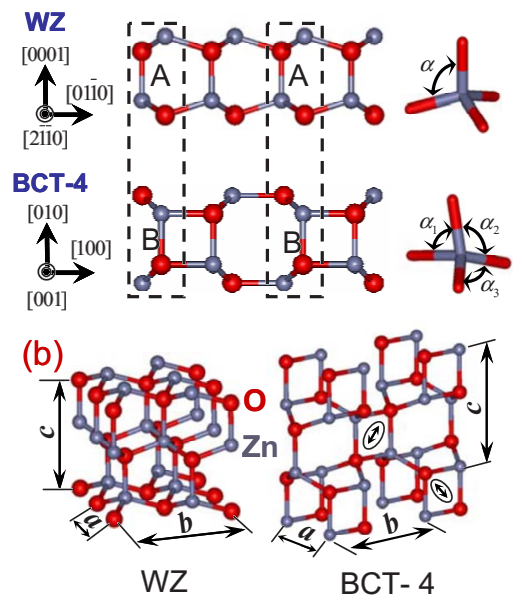

FIG. 2. (Color online) (a) Wurtzite (WZ) and body-centeredtetragonal with four-atom rings (BCT-4) structures and (b) crystallographic transition through breaking and formation of bonds and differences in bond angles between the WZ and the BCT-4 structures.

nanorods analyzed here have the same length of $145.8 \AA$ and five different cross-sectional widths $(d=19.5,26.0,32.5$, 39.0, and $45.5 \AA$ ). A Buckingham-type potential with charge interactions is used to define atomic interactions in the MD calculations. ${ }^{13,17,18}$ The analysis concerns quasistatic deformation at $300 \mathrm{~K} .^{1}$

Figure 1(b) shows the stress-strain response of a nanorod with lateral dimension $d=32.5 \AA$. Four distinct stages are observed. The first stage $(\mathrm{A} \rightarrow \mathrm{B})$ corresponds to the elastic stretching of the wurtzite (WZ) structure up to a strain of $7.5 \%$. Further deformation results in a precipitous stress drop $(\mathrm{B} \rightarrow \mathrm{C})$ associated with the WZ to BCT-4 phase transformation. The transformation completes at a strain of $8.5 \%$. Continued loading causes elastic stretching of the BCT-4 structure $(\mathrm{C} \rightarrow \mathrm{D})$ and culminates in the eventual failure at a strain of $16.9 \%$ (point E). To analyze the stability of the parent and transformed structures, unloading is performed from states prior to transformation initiation (first peak tensile stress, point B) and failure initiation of the nanorod (second peak tensile stress, point $\mathrm{D}$ ). The unloading path from $\mathrm{B}$ coincides with the loading path, confirming that the deformation from A to B is indeed the elastic response of the WZ-structured nanorod. Unloading from $\mathrm{D}$ also results in the elastic recovery of the BCT-4 structure, and continued unloading beyond the transformation completion strain (point $\mathrm{C}$ ) does not result in a reverse transformation. Instead, the nanorod retains the BCT-4 structure when the stress is reduced to zero [F in Fig. 1(b)].

The WZ to BCT-4 transformation occurs through a combination of (1) the breaking of every other $\mathrm{Zn}-\mathrm{O}$ bond along the [0001] direction [bond A in Fig. 2(a)] and (2) the formation of an equal number of $\mathrm{Zn}-\mathrm{O}$ bonds along the same direction [bond B in Fig. 2(a)] next to the broken bonds. This process repeats on alternate planes along the [0110] direction. The transformed structure retains the tetrahedral coordination with each $\mathrm{Zn} / \mathrm{O}$ atom at the center and four $\mathrm{O} / \mathrm{Zn}$
TABLE I. Lattice constants for WZ and BCT-4 ZnO in tension along the $c$ axis obtained via MD and DFT (in square brackets) calculations.

\begin{tabular}{|c|c|c|c|c|c|}
\hline \multirow[b]{2}{*}{ Parameters } & \multirow{2}{*}{$\begin{array}{l}\mathrm{WZ} \\
\sigma=0\end{array}$} & \multicolumn{4}{|c|}{ BCT-4 } \\
\hline & & $\sigma=0$ & $\sigma=4$ & $\sigma=7$ & $\sigma=10$ \\
\hline$a(\AA)$ & $\begin{array}{c}3.29 \\
{[3.20]}\end{array}$ & $\begin{array}{c}3.24 \\
{[3.17]}\end{array}$ & $\begin{array}{c}3.22 \\
{[3.13]}\end{array}$ & $\begin{array}{c}3.20 \\
{[3.09]}\end{array}$ & $\begin{array}{c}3.19 \\
{[3.06]}\end{array}$ \\
\hline$b(\AA)$ & $\begin{array}{c}5.67 \\
{[5.55]}\end{array}$ & $\begin{array}{c}5.58 \\
{[5.48]}\end{array}$ & $\begin{array}{c}5.54 \\
{[5.42]}\end{array}$ & $\begin{array}{c}5.51 \\
{[5.35]}\end{array}$ & $\begin{array}{c}5.48 \\
{[5.32]}\end{array}$ \\
\hline$c(\AA)$ & $\begin{array}{c}5.17 \\
{[5.13]}\end{array}$ & $\begin{array}{c}5.52 \\
{[5.48]}\end{array}$ & $\begin{array}{c}5.67 \\
{[5.71]}\end{array}$ & $\begin{array}{c}5.77 \\
{[5.87]}\end{array}$ & $\begin{array}{c}5.84 \\
{[5.98]}\end{array}$ \\
\hline$V=a b c\left(\AA^{3}\right)$ & $\begin{array}{c}96.4 \\
{[91.1]}\end{array}$ & $\begin{array}{c}99.8 \\
{[95.2]}\end{array}$ & $\begin{array}{c}101.2 \\
{[96.9]}\end{array}$ & $\begin{array}{c}101.7 \\
{[97.0]}\end{array}$ & $\begin{array}{l}102.1 \\
{[97.3]}\end{array}$ \\
\hline$\Delta V\left(\AA^{3}\right)$ & $\begin{array}{c}0.0 \\
{[0.0]}\end{array}$ & $\begin{array}{c}3.4 \\
{[4.1]}\end{array}$ & $\begin{array}{c}4.8 \\
{[5.8]}\end{array}$ & $\begin{array}{c}5.3 \\
{[5.9]}\end{array}$ & $\begin{array}{c}5.7 \\
{[6.2]}\end{array}$ \\
\hline$c / a$ & $\begin{array}{c}1.57 \\
{[1.60]}\end{array}$ & $\begin{array}{c}1.71 \\
{[1.73]}\end{array}$ & $\begin{array}{c}1.76 \\
{[1.82]}\end{array}$ & $\begin{array}{c}1.80 \\
{[1.9]}\end{array}$ & $\begin{array}{c}1.83 \\
{[1.95]}\end{array}$ \\
\hline$b / a$ & $\begin{array}{c}1.72 \\
{[1.73]}\end{array}$ & $\begin{array}{c}1.72 \\
{[1.73]}\end{array}$ & $\begin{array}{c}1.72 \\
{[1.73]}\end{array}$ & $\begin{array}{c}1.72 \\
{[1.73]}\end{array}$ & $\begin{array}{c}1.71 \\
{[1.73]}\end{array}$ \\
\hline
\end{tabular}

atoms are at the vertices of a tetrahedron. The geometry of the tetrahedron can be characterized through the O-Zn-O bond angles $\left(\alpha_{i}, i=1-6\right)$, as shown in Fig. 2(a). For WZ, all bond angles are approximately equal $\left(\alpha_{i} \approx 108^{\circ}\right)$. For BCT-4, the formation of four-atom rings results in three distinct bond angles $\left(\alpha_{1} \approx 90^{\circ}, \alpha_{2} \approx 112.7^{\circ}\right.$, and $\left.\alpha_{3} \approx 113.7^{\circ}\right)$.

As seen from Fig. 2(b), the transformed phase consists of four-atom (two $\mathrm{Zn}$ and two $\mathrm{O}$ ) rings arranged in a $\mathrm{BCT}$ lattice. Note that the four-atom ring at the center is rotated by $90^{\circ}$ relative to the rings at the corners of the tetragonal lattice cell. Strictly speaking, the unit cell consists of two-ring clusters (one of each orientation, total of eight atoms) positioned in a simple tetragonal primitive lattice. Figure 2(b) also shows the lattice parameters $a, b$, and $c$ for the WZ and BCT-4 structures. Their respective values as obtained from MD and DFT calculations (in square brackets) at various stress levels are listed in Table I along with unit cell volumes. For WZ, the ratios $c / a$ and $b / a$ are 1.60 and 1.73 , respectively. Throughout the transformation, the $b / a$ ratio remains at its initial value of $1.73( \pm 0.02)$, reflecting the symmetries of the loading and the lattice. On the other hand, upon transformation to BCT-4 at a stress above $7 \mathrm{GPa}$, the $c / a$ ratio increases to 1.8 . Phenomenologically, the predilection for the BCT-4 phase over the WZ phase under the tensile loading conditions considered here can be explicated by its elongated configuration in the [0001] direction (higher $c / a$ ratio) relative to that of the WZ structure. Upon unloading, the residual strain at F in Fig. 1(b) is $6.8 \%$ according to both $\mathrm{MD}$ and DFT. It reflects the dimensional difference between the unstressed WZ and BCT-4 structures in the [0001] direction. This unstressed BCT-4 structure corresponds to the "ideal" BCT-4 structure predicted by the DFT calculations with $b / a=c / a=1.73$ in Fig. 3(a).

The relative favorability of the two phases is studied by calculating the enthalpy (per four $\mathrm{Zn}-\mathrm{O}$ pairs) using DFT calculations. ${ }^{2,19}$ The complete enthalpy surfaces (not shown 

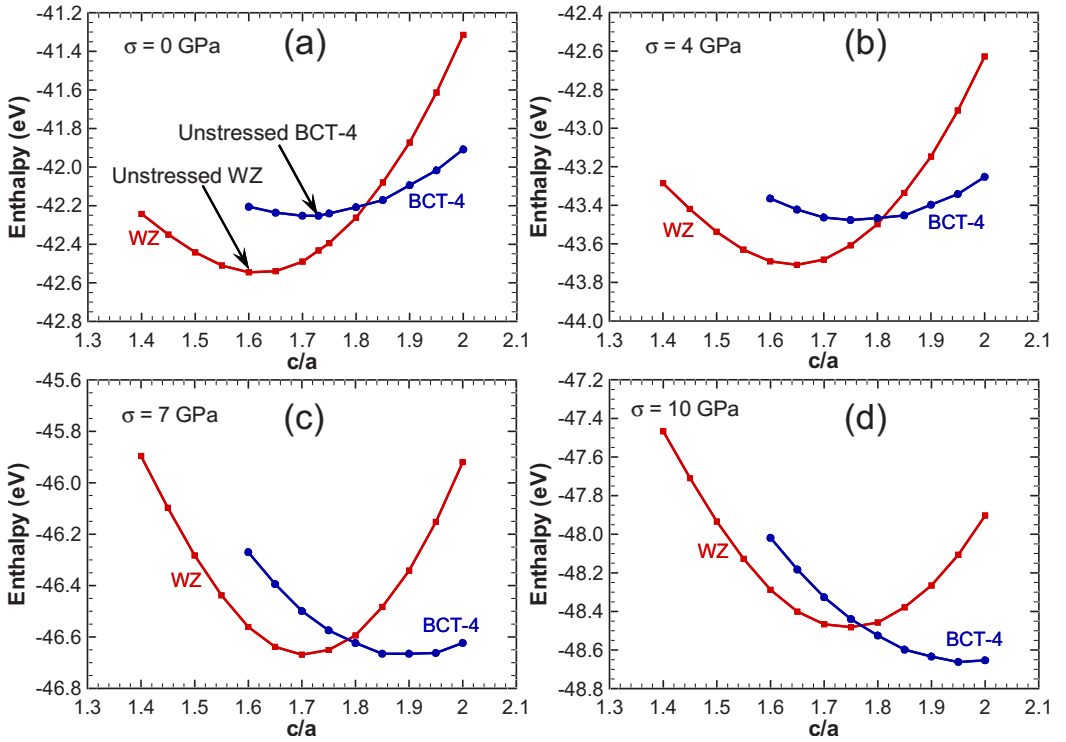

FIG. 3. (Color online) Enthalpy (per four $\mathrm{Zn}-\mathrm{O}$ pairs) as a function of $c / a$ obtained from DFT calculations for $b / a=1.73$ at tensile stresses of (a) $\sigma=0 \mathrm{GPa}$, (b) $\sigma=4 \mathrm{GPa}$, (c) $\sigma=7 \mathrm{GPa}$, and (d) $\sigma=10 \mathrm{GPa}$ in the [0001] direction. due to space limitation) show that the BCT-4 structure has minimum enthalpy at $b / a=1.73$ for all values of tensile stress considered. For clarity without loss of generality, the discussions here use Fig. 3 which shows the enthalpy values (eV per four $\mathrm{Zn}-\mathrm{O}$ pairs) for both structures for $b / a=1.73$ at $\sigma=0,4,7$, and $10 \mathrm{GPa}$. At any stress level, each structure has its own enthalpy minimum. The first minimum is in the vicinity of $c / a \approx 1.6$ which corresponds to WZ with lattice parameters slightly different from those at zero stress, and the second minimum is in the vicinity of $c / a \approx 1.7-1.9$, which corresponds to BCT-4. At zero stress, WZ is the stable crystal structure and its enthalpy is lower than that of BCT-4 by $0.3 \mathrm{eV}$ [Fig. 3(a)]. As the stress is increased to $4 \mathrm{GPa}$ [Fig. 3(b)], the difference in enthalpies decreases, and at a stress of $7 \mathrm{GPa}$ [Fig. 3(c)], the two minima become comparable indicating that $\mathrm{WZ}$ and BCT-4 are equally favored. This value of stress corresponds to the equilibrium transition stress for the two phases. Since an energy barrier (associated with intermediate transitional states) exists for the transformation, a stress level higher than the $7 \mathrm{GPa}$ equilibrium stress is required to initiate the transformation. At a stress of $10 \mathrm{GPa}$ [Fig. 3(d)], the enthalpy of BCT-4 is lower, and this structure is clearly favored. Further increases in stress result in the eventual initiation of the phase transformation. The specific stress level at which the transformation initiates depends on the rod size and temperature. For the particular nanorod in Fig. 1 at $300 \mathrm{~K}$, the critical stress level is $\sigma$ $=17.9 \mathrm{GPa}$. The gradual evolution of the local enthalpy minimum for the BCT-4 at $\sigma=0$ into a global minimum as stress increases confirms that the phase transformation observed in MD simulations is indeed energetically favored.

The phase transformation observed here alters the electrical, thermal, and mechanical responses of the nanorods. Recently, WZ-structured $\mathrm{ZnO}$ nanorods have been used to successfully generate direct electric current through mechanical bending. ${ }^{20}$ The transformation from the piezoelectric WZ structure to the nonpiezoelectric BCT-4 structure establishes an upper bound for the maximum possible current generation and operational strain for this application. Specifically, the electric field output $E_{3}$ can be related to the longitudinal strain $\varepsilon_{3}$ through $E_{3}=\varepsilon_{3} / d_{33}$, where $d_{33} \approx 20.5 \mathrm{pm} / \mathrm{V}$ is the piezoelectric coefficient for the $\mathrm{ZnO}$ nanorods. Since the strain at the initiation of transformation [B in Fig. 1(b)] is approximately $7.5 \%$ for all rod sizes, the maximum electric field output is therefore $3.7 \mathrm{~V} / \mathrm{nm}$. The mechanical response of BCT-4 also differs significantly from that of WZ. In particular, the enthalpy curves for BCT-4 are flatter than those for WZ (Fig. 3), indicating that the elastic stiffness of BCT-4 is lower than that for WZ. Indeed, in Fig. 1(b), the slope of curve AB (228 GPa, which is the [0001] elastic modulus of WZ) is higher than that of curve FD (167 GPa, which is the corresponding modulus of BCT-4). The thermal response of semiconductors such as $\mathrm{ZnO}$ is dominated by phonons and the interactions between phonons and surfaces. ${ }^{21}$ The WZ to BCT-4 phase transformation changes the atomic arrangement and hence the phonon spectrum, resulting in potentially large changes in thermal conductivity. The electronic band structures of WZ and BCT-4 are shown in Fig. 4. Note that the total number of bands for BCT-4 is twice that for WZ because the unit cell of BCT-4 has twice as many atoms as WZ. Both phases have direct band gaps at $\Gamma$. Although DFT calculations with local density approximations are known to underestimate band gaps and therefore are not normally used to predict absolute band gap values, they can provide valid (a) WZ

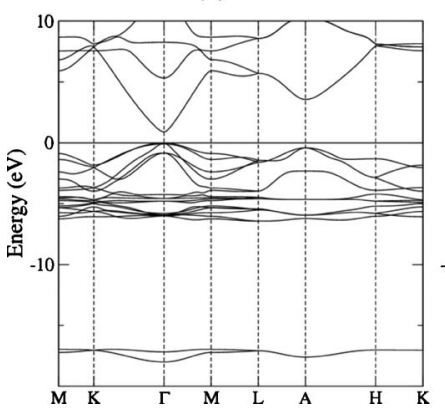

(b) $\mathrm{BCT}-4$

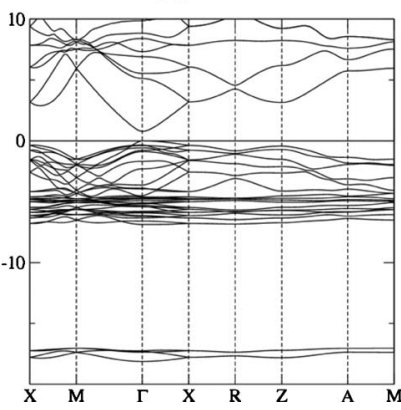

FIG. 4. Band structures of (a) $\mathrm{WZ} \mathrm{ZnO}$ and (b) BCT-4 $\mathrm{ZnO}$ obtained by DFT calculations. The energy is relative to the top of the valence bands. 


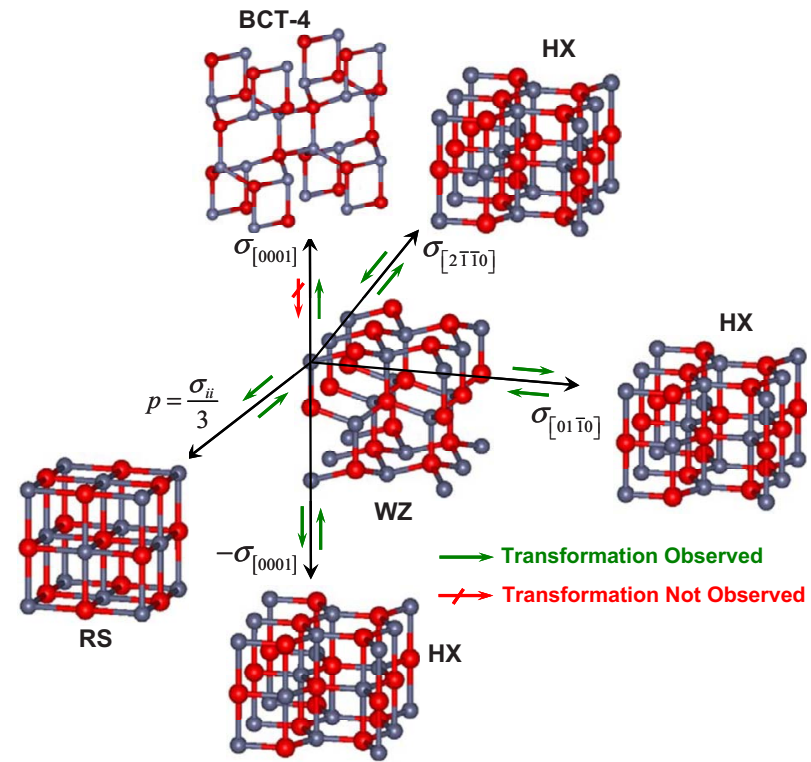

FIG. 5. (Color online) Crystalline structure-load triaxiality map summarizing the nature and much wider extent of polymorphism in $\mathrm{ZnO}$ than previously known; WZ is the natural state at ambient conditions, RS occurs under hydrostatic or near hydrostatic compression, HX occurs under tension along the [2 $\overline{1} \overline{1} 0]$ and [01 $\overline{1} 0]$ directions as well as compression along the [0001] direction, and BCT-4 occurs under tension along the [0001] direction. The green and red arrows indicate, respectively, possible and impossible transformation paths under relevant load direction reversals. ZB can only be grown epitaxially on certain crystalline planes of cubic crystals and cannot be obtained via a transformation from WZ under external loading; therefore, it is not included in this map. relative comparisons between the two phases. The calculated band gap and average electron effective mass of BCT-4 are, respectively, $12 \%$ and $17 \%$ smaller than those of $\mathrm{WZ}$, giving the nanorod a smaller band gap and potentially higher electron mobility after the WZ-to-BCT-4 transformation. These mechanically induced electrical property shifts may have applications in devices that depend on coupling between responses.

Most importantly, the identification of the BCT- 4 structure leads to a more complete understanding of the nature and extent of polymorphism in $\mathrm{ZnO}$ and its dependence on load triaxiality. Joining wurtzite (WZ), zinc blende (ZB), rocksalt (RS), and HX, BCT-4 constitutes the fifth polymorph of $\mathrm{ZnO}$ discovered so far. It is now possible to construct a structureload triaxiality map for $\mathrm{ZnO}$, as shown in Fig. 5. Among the previously well known phases, WZ is the most stable and naturally occurring phase and RS is observed under hydrostatic compressive conditions. Both BCT-4 and HX are stabilized under uniaxial loading, with HX occurring under tension along the $[01 \overline{1} 0]$ and/or [2 $\overline{1} \overline{1} 0]$ directions as well as compression along the [0001] direction and BCT-4 occurring under tension along the [0001] direction. It is worthwhile to note that ZB grows epitaxially on specific surfaces of cubic crystals and cannot be obtained via a transformation from WZ under external loading; therefore, it is not included in this map.

Support from NSF (CMS9984298), NSFC (10772012, 10432050, and 10528205), NANOTEC (NN49-024), and TRF (BRG4880015 and PHD/0264/2545) is acknowledged. Computations are carried out at the NAVO, ARL, ASC MSRCs, NSRC, and the LSEC of the CAS.
*Author to whom correspondence should be addressed; FAX: 404894-0186; min.zhou@gatech.edu

${ }^{1}$ A. J. Kulkarni, K. Sarasamak, S. Limpijumnong, and M. Zhou, Philos. Mag. 87, 2117 (2007).

${ }^{2}$ A. J. Kulkarni, M. Zhou, K. Sarasamak, and S. Limpijumnong, Phys. Rev. Lett. 97, 105502 (2006).

${ }^{3}$ L. Zhang and H. Huang, Appl. Phys. Lett. 89, 183111 (2006).

${ }^{4}$ L. Zhang and H. Huang, Appl. Phys. Lett. 90, 023115 (2007).

${ }^{5}$ P. A. Schultz and E. B. Stechel, Phys. Rev. B 57, 3295 (1998).

${ }^{6}$ M. Marezio, Acta Crystallogr. 19, 396 (1965).

${ }^{7}$ M. Marezio and J. P. Remeika, J. Chem. Phys. 44, 3348 (1966).

${ }^{8}$ M. S. Arnold, P. Avouris, Z. W. Pan, and Z. L. Wang, J. Phys. Chem. 107, 659 (2003).

${ }^{9}$ X. D. Bai, P. X. Gao, Z. L. Wang, and W. G. Wang, Appl. Phys. Lett. 82, 4806 (2003).

${ }^{10}$ E. Comini, G. Faglia, G. Sberveglieri, Z. W. Pan, and Z. L. Wang, Appl. Phys. Lett. 81, 1869 (2002).

${ }^{11}$ Z. L. Wang and J. Song, Science 312, 242 (2006).
${ }^{12}$ J. Diao, K. Gall, and M. L. Dunn, Phys. Rev. B 70, 075413 (2004).

${ }^{13}$ A. J. Kulkarni, M. Zhou, and F. J. Ke, Nanotechnology 16, 2749 (2005).

${ }^{14}$ W. Liang and M. Zhou, Phys. Rev. B 73, 115409 (2006).

${ }^{15}$ A. Umar, B. Karunagaran, E. K. Suh, and Y. B. Hahn, Nanotechnology 17, 4072 (2006).

${ }^{16}$ A. Wei, X. W. Sun, C. X. Xu, Z. L. Dong, Y. Yang, S. T. Tan, and W. Huang, Nanotechnology 17, 1740 (2006).

${ }^{17}$ D. J. Binks and R. W. Grimes, J. Am. Ceram. Soc. 76, 2370 (1993).

${ }^{18}$ A. J. Kulkarni and M. Zhou, Acta Mech. Sin. 22, 217 (2006).

${ }^{19}$ S. Limpijumnong and S. Jungthawan, Phys. Rev. B 70, 054104 (2004).

${ }^{20}$ M. H. Zhao, Z. L. Wang, and S. X. Mao, Nano Lett. 4, 587 (2004).

${ }^{21}$ A. J. Kulkarni and M. Zhou, Appl. Phys. Lett. 88, 141921 (2006). 